\title{
SISTEMA PARA LA EVALUACIÓN DE CAPACIDADES DE INNOVACIÓN EN PYMES DE PAÍSES EN DESARROLLO: CASO PANAMÁ*
}

\author{
SIDIA MORENO ROJAS** \& AGUEDA GARCÍA CARRILLO**** \\ UNIVERSIDAD POLITÉCNICA DE CATALUÑA (ESPAÑA)
}

Recibido/ Received/ Recebido: 10/12/2013 - Aceptado/ Accepted / Aprovado: 29/05/2014

\begin{abstract}
Resumen
El objetivo de la investigación ha sido generar un sistema de evaluación de capacidades de innovación aplicable a pequeñas y medianas empresas (PYMES), de tal manera que se pueda conocer a fondo las capacidades de innovación en una organización permitiendo identificar falencias y requerimientos fundamentales para su éxito. La literatura converge hacia una doble dimensión al estudiar la innovación: como sistema y como proceso; donde las consideraciones contextuales son fundamentales. Para generar el sistema se investigaron enfoques, modelos, herramientas y pautas metodológicas de innovación. Se analizaron las características de procesos desde el punto de vista los países en desarrollo (PED), se compararon funciones de gestión de innovación y se establecieron interrelaciones entre funciones y variables del sistema. Como resultado se determinaron cinco capacidades endógenas requeridas para innovar y sus correspondientes categorías de análisis, así como dos nuevos elementos diferenciadores de procesos de innovación en PED: los focos de procesos de aprendizaje (FPA) y los focos de innovaciones incrementales (FII). Los resultados han sido aplicados en cuatro casos de PYMES en Panamá, concluyendo que la evaluación de capacidades permite situar a las organizaciones en diferentes niveles, compararlas e identificar falencias y posibles hojas de ruta para impulsar procesos de innovación.
\end{abstract}

Palabras clave: Sistema de evaluación, Capacidades de innovación, Capacidad endógena, PYMES, Proceso de aprendizaje.

* El artículo es producto de los resultados de la tesis doctoral: Moreno, S. (2013). Sistema para la gestión de innovación en países en desarrollo, basado en la autoevaluación integrada de capacidades endógenas. Articulación de la base conceptual del sistema experto para la autoevaluación. Ph. D. Thesis. Barcelona: Univesitat Politecnica de Catalunya.

** Doctorado en Ingeniería de Proyectos de la Universidad Politécnica de Cataluña (UPC). Investigadora, coordinadora de servicios especializados del Centro de Investigaciones e Innovación Eléctrica, Mecánica y de la Industria (CINEMI). Grupo de investigación: Gestión de Innovación y Operaciones Empresariales - CINEMI, Universidad Tecnológica de Panamá (UTP). Correo electrónico: sidia. moreno@utp.ac.pa. Tel: 5072908400 ext. 8461 (Panamá). Dirección postal: Departamento de Proyectos de Ingeniería, Av. Diagonal 647, ETSEIB planta 10, 08290 Barcelona (España).

*** Doctora en Ciencias Químicas dentro del programa de Ingeniería de Sistemas Técnicos (UPC), Máster de Ergonomía (UPC). Postgrado Fase creativa en la innovación de los productos y servicios (UPC). Licenciada en Química de la Universidad Ramón Llull. Ingeniera química IQS, Instituto Químico de Sarriá (centro de I+D). Investigadora, docente, coordinadora (UPC). Grupo de investigación: GIIP - Grupo de Investigación en Ingeniería de Proyectos: Diseño, Sostenibilidad y Comunicación. Correo electrónico: agueda.garcia@ upc.edu. Teléfono: +34 934016640 (España). Dirección postal: Departamento de Proyectos de Ingeniería, Av. Diagonal 647, ETSEIB planta 10, 08290 Barcelona (España). 


\title{
INNOVATION CAPACITY ASSESSMENT SYSTEM IN SMALL AND MEDIUM ENTERPRISES IN DEVELOPING COUNTRIES: CASE OF PANAMA
}

\begin{abstract}
The aim of this research was to generate an evaluation system of innovation capabilities for small and medium enterprises (SMEs), in order to know innovation capabilities in an organization to identify gaps and fundamental requirements for success. Literature converges in two dimensions to consider innovation: as a system and as a process; where contextual considerations are critical. In order to build the system approaches, models, tools and methodological innovation patterns were investigated. Process characteristics were analyzed for developing countries (DCs), innovation management functions were compared and relationships between functions and system variables were established. As result, five endogenous capabilities required to innovate were determined with their corresponding analysis categories as wells as, two new differentiation elements of innovation processes in PED: learning processes Outbreaks (FPA because of its acronym in Spanish) and incremental innovation outbreaks (FII because of its acronym in Spanish). Results have been applied in four cases of SMEs in Panama, concluding that capability assessment allows organizations to pace on different levels, compare and identify possible gaps and roadmaps to drive innovation processes. Keywords: Assessment system, Innovation capabilities, Endogenous capabilities, SMEs, Learning process.
\end{abstract}

\section{SISTEMA PARA A AVALIAÇÃO DE CAPACIDADES DE INOVAÇÃO EM PEQUENAS E MÉDIAS EMPRESAS DE PAÍSES EM DESENVOLVIMENTO: CASO PANAMÁ}

\section{Resumo}

O objetivo da pesquisa foi gerar um sistema de avaliação de capacidades de inovação aplicável a pequenas e médias empresas (PYMES), de tal maneira que se possa conhecer a fundo as capacidades de inovação numa organização permitindo identificar falhas e necessidades fundamentais para seu sucesso. A literatura converge para uma dupla dimensão ao estudar a inovação: como sistema e como processo, onde as considerações contextuais são fundamentais. Para gerar o sistema se pesquisaram enfoques, modelos, ferramentas e pautas metodológicas de inovação. Analisaramse as características de processos desde o ponto de vista dos países em desenvolvimento (PED), compararam-se funções de gestão de inovação e se estabeleceram inter-relações entre funções $e$ variáveis do sistema. Como resultado se determinaram cinco capacidades endógenas requeridas para inovar e suas correspondentes categorias de análises, bem como, dois novos elementos diferenciadores de processos de inovação em PED: os focos de processos de aprendizagem (FPA) e os focos de inovações incrementais (FII). Os resultados foram aplicados em quatro casos de PYMES no Panamá, concluindo que a avaliação de capacidades permite situar às organizações em diferentes níveis, compará-las e identificar falhas e possíveis rotas para impulsionar processos de inovação.

Palavras chave: Sistema de avaliação, Capacidades de inovação, Capacidade endógena, PYMES, Processo de aprendizagem.

Moreno, S. \& García, A. (2014) Sistema para la Evaluación de Capacidades de Innovación en PYMES de Países en Desarrollo: Caso Panamá. En: Revista de la Facultad de Ciencias Económicas de la Universidad Militar Nueva Granada. rev.fac.cienc.econ, XXII (2).

JEL: O31, O32, O39. 


\section{Introducción}

En el análisis de la innovación, la literatura converge hacia una doble dimensión: como sistema (Schumpeter, 1939; Nelson, 1993) y como proceso (Lundvall, 1992; Nelson, 1997); donde las consideraciones contextuales son fundamentales.

La innovación como sistema enfatiza el papel de las interacciones entre los agentes involucrados y el aprendizaje generado por éstas, activando flujos de conocimiento multidireccionales que tendrían que ser reconocidos y aprovechados por las empresas para mejorar su competitividad, lo que implica contar con capacidades específicas, las cuales son necesarias identificar.

La innovación como proceso aborda las características de cómo se lleva a cabo la misma y las capacidades inherentes para realizar las acciones requeridas. El proceso se ha de entender como la transformación de una idea en un producto vendible (nuevo o mejorado); o proceso operativo en la industria o en el comercio; o bien, nuevo método de servicio o cambio organizacional. Este proceso requiere de decisiones y acciones en las que las capacidades para innovar de la organización son determinantes, siendo éstas influenciadas por el entorno.

Al respecto, Porter (1998, 77-90) hace énfasis en la visión de una organización de aprendizaje interacti$v o$, en donde la existencia de capacidades internas se concreta en prácticas institucionalizadas. Una de estas capacidades internas (o endógenas), es la capacidad de innovación (CI). Hidalgo, León \& Pavón (2000) argumentan que la CI es un recurso fundamental para una empresa y que para aprovechar las oportunidades en el entorno se requiere de estrategias empresariales que estarán condicionadas por ésta capacidad.

Las preguntas de investigación que se plantean son: ¿cómo identificar y medir ésta CI en una empresa?, ¿qué requieren las empresas para contar con $\mathrm{Cl}$ ?, ¿qué componentes o factores determinan la $\mathrm{Cl}$ ?, ¿cómo podrían ser articulados en un sistema consistente y funcional estos componentes o factores de la $\mathrm{Cl}$ ?, ¿es posible que a partir de la evaluación de la $\mathrm{CI}$ en una empresa se pueda generar una hoja de ruta para el fortalecimiento de la misma?

La investigación que se presenta permitió identificar componentes y factores de la $\mathrm{CI}$ a los cuales ha denominado capacidades endógenas. Para lo cual se realizó un análisis de modelos de evaluación de innovación existentes, se identificaron variables $e$ indicadores y se establecieron relaciones entre tales capacidades; lográndose un fundamento conceptual y metodológico para la evaluación. Los resultados obtenidos han permitido generar una primera versión de un sistema de evaluación de capacidades de innovación (SIGE V1), basado en capacidades endógenas, y aplicable a PYMES de PED.

\section{Metodología y fases de la investigación}

Se trata de una investigación exploratoria de ciencia aplicada, basada en el análisis documental y la verificación de resultados mediante casos de estudio que fueron seleccionados por conveniencia por lo complicado de contar con PYME en actividades investigativas.

La investigación se realizó en cuatro etapas: (i) revisión de literatura; (ii) generación del modelo conceptual de Gestión de Innovación (GI) e identificación de capacidades; (iii) determinación de componentes del SIGE V1, los descriptores para evaluar las capacidades; y (iv) verificación del sistema mediante cuatro estudios de casos.

Para generar el SIGE V1 se investigaron y analizaron enfoques, modelos, herramientas y pautas metodológicas de innovación. Se analizaron las características de procesos desde el punto de vista de PED, se compararon funciones de gestión de innovación $y$ se establecieron interrelaciones entre funciones $y$ variables del sistema.

\section{Revisión de literatura sobre evaluación de capacidades endógenas}

De acuerdo con el diccionario de la Real Academia (RAE, 1992, 654) la evaluación es la acción y el efecto de estimar, apreciar, calcular el valor de una cosa, para ello se debe establecer el proceso de medir de 
manera cuantitativa o determinar de manera cualitativa un estado actual con respecto a un estado inicial o referencia.

Al hacer referencia a un sistema de evaluación en la presente investigación, se está considerando el concepto de un sistema abierto, el cual según la Teoría de Sistemas, posee cuatro componentes fundamentales: entrada, proceso, salida y retro-alimentación (Gráfica 1).

Gráfica 1. Sistema de evaluación ${ }^{1}$

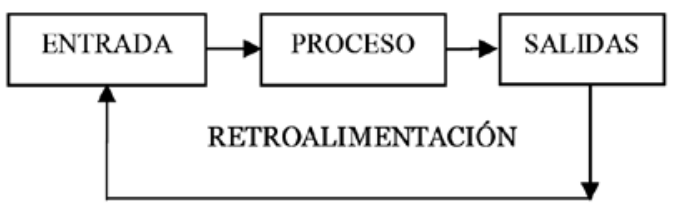

Las herramientas y metodologías que por lo general se utilizan para evaluar la innovación son insuficientes y en su mayor parte, corresponden a métodos basados en la cientometría que consiste en el diseño y utilización de indicadores de ciencia y tecnología (CyT), para la comparación entre países y regiones (nivel supranacional). Se utilizan también métodos descriptivos enfocados en procesos o resultados de innovación a nivel de país o región, más no así de manera específica a lo interno de las empresas.

Dentro de este contexto, parece correcto concebir el sistema de evaluación de $\mathrm{CI}$ de la siguiente manera: las entradas constituyen la información inicial de la empresa de acuerdo a los indicadores o descriptores, el proceso constituye la aplicación de la métrica y los procedimientos establecidos y las salidas se refieren a los resultados de cada indicador, la posible hoja de ruta y los nuevos conocimientos generados durante el proceso (tácitos o codificados); la retroalimentación da lugar a nuevas entradas de información y de conocimientos que fortalecen y dinamizan el proceso de evaluación.

El conocimiento puede ser el incorporado en los procedimientos, manuales de tecnología, procesos habituales y otros medios de la empresa, denominado conocimiento codificado o, el incorporado esencialmente en el cerebro de las personas, denominado conocimiento tácito, el cual presenta numerosas dificultades al intentar medirlo (OCDE, 2005, 25).

Hidalgo, León \& Pavón (2000) plantean que la Capacidad de Innovación $(\mathrm{CI})$, constituye un recurso importante de la empresa al igual que sus capacidades financieras, comerciales y productivas, y que para aprovechar las oportunidades latentes en el entorno se requiere de estrategias empresariales que estarán condicionadas por dicha capacidad, lo que hace referencia al enfoque estratégico de la evaluación de innovación.

El Manual de Bogotá (Jaramillo, Lugones \& Salazar, 2001, 33) define la CI como: la potencialidad de idear, planear y realizar innovaciones del tipo que sea a partir del uso de los conocimientos tecnológicos y organizativos formales e informales presentes en la empresa y/o contratados externamente en el mercado. En esta definición la CI está en función, en parte, de la aplicación del conocimiento tecnológico interno o adquirido.

Por su parte, Yoguel \& Boscherini (1996) plantean que para desarrollar la $\mathrm{CI}$ se requiere un umbral de competencias que pueden verse afectadas por factores como el sendero evolutivo tecnológico, capacidad de aprender y grado de desarrollo del ambiente en que actúan las empresas (entorno). Lo que denota que existen factores determinantes de la $\mathrm{CI}$, que van más allá de la posibilidad de aplicación de conocimientos tecnológicos, a los cuales habrá que ponerle singular atención.

El concepto de capacidad endógena que se enfoca en la presente investigación, hace alusión a lo que Porter (1998) denominó "capacidades internas", con lo cual se amplía el enfoque en los procesos de evaluación de innovación, en que se ha de considerar la CI como un conjunto de capacidades endógenas o internas que han sido consideradas de manera específica por los diferentes autores que a continuación son descritos.

1 Fuente: Elaboración propia. 
Lundvall (1992) afirma que "el conocimiento es el recurso más importante en la economía moderna, y qué el aprendizaje es el proceso más destacable, ya que su valor no decrece con el uso, no es un bien escaso, posee elementos tácitos difíciles de transmitir, y se encuentra en un contexto donde los fallos del mercado son la regla, en lugar de la excepción". Plantea además que el aprendizaje requiere de una capacidad que en la mayoría de los casos se deriva de la experiencia, es decir, del aprender-haciendo, aprender-usando y aprender-interactuando.

Al respecto, en el enfoque evolucionista se enfatiza el papel central de las interacciones entre agentes en procesos de aprendizajes, en función de los flujos de conocimiento (Jaramillo et al., 2001, 32; Pavitt, 1991).

Todo lo anterior permite identificar como fundamental en los procesos de innovación la Capacidad de Aprendizaje (CA), que se ha de entender como la capacidad endógena de una empresa para adquirir, analizar, aplicar y transformar conocimiento.

De manera complementaria surge una nueva generación de estudios que abordan la denominada Capacidad Tecnológica (CT), relacionada con el impacto de los procesos de aprendizaje en la trayectoria de acumulación de conocimientos tecnológicos de empresas en PED (Figueiredo, 2001, 289-290). Este sentido se permite examinar la base organizacional de los procesos de aprendizaje y sus consecuencias en la acumulación de los mismos.

Lall (1992) y Bell \& Pavitt (1995) abordan la CT, como aquella capacidad que incorpora todos los recursos necesarios para generar y administrar los cambios tecnológicos. Tales recursos se acumulan y almacenan en los individuos (actitudes, conocimiento y experiencia) y en los sistemas organizacionales. Lo que permite distinguir dos tipos de recursos: (i) aquellos que son necesarios para usar los sistemas de producción existentes y (ii) los que son necesarios para cambiar los sistemas de producción.

El desarrollo de CT en las empresas de PED exige una complementación entre las informaciones y el know-how que aportan las tecnologías generadas en los países desarrollados (PD) y la acumulación de aprendizajes a partir de los esfuerzos endógenos realizados por los PED. Lo cual implica que el no realizar actividades locales de $\mathrm{I}+\mathrm{D}$ puede conllevar para las empresas de los PED esfuerzos adversos, que podría reflejarse en una dependencia tecnológica ineficaz. Para contrarrestar esta situación los vínculos formales con universidades $\mathrm{y} / \mathrm{o}$ centros de $\mathrm{I}+\mathrm{D}$ nacionales o internacionales se constituye en una fortaleza.

Para analizar y explicar más a fondo lo concerniente al desarrollo de la CT y queriendo establecer la no interdependencia con otras capacidades de rutina (administrativas, financieras, etc.), Lall (1992) desarrolla un modelo en el cual las CT de una empresa son categorizadas en funciones tecnológicas: decisión y control sobre la planta; ingeniería de proyectos; procesos y organización de la producción, productos y equipos. Basados en estas funciones, se plantea que es posible que una empresa acumule ciertas capacidades innovadoras sin que la acumulación de sus capacidades de rutina se encuentre totalmente consolidada. Este fenómeno es conocido como de acumulación truncada o incompleta, y es observado con cierta frecuencia en empresas que operan en economías en desarrollo (Figueiredo, 2001, 289-290).

Por otro lado, Cohen \& Levinthal (1990), abordan otro tipo de capacidad, la Capacidad de Asimilación de Tecnología (CAT), definida como la habilidad de la empresa para reconocer, internalizar y explotar el conocimiento tecnológico externo y generar valor en la empresa en forma de nuevos productos o servicios. La asimilación de tecnología guarda estrecha relación con actividades de la gestión tecnológica y de innovación; debido a que asimilan los conocimientos de cada tecnología adquirida por la empresa, genera un proceso de aprendizaje virtuoso capaz de propiciar un ambiente innovador (OCDE, 2005, 29; Castro et al., 2009).

En la práctica los resultados en la región latinoamericana han demostrado que tal proceso de aprendizaje no se produce a satisfacción (Angelelli \& Gligo, 2002; REDES \& RICYT, 2008; CEPAL, 2010; Dini, 2004), sobre todo por estar severamente condicionado por la falta de acumulación de aprendizajes y la no existencia de estructuras formales en los procesos de adopción de tecnología. 
Otra capacidad endógena es la que tiene que ver con las relaciones, vínculos y complementariedades que una empresa establece con otros agentes del entorno. Estas pueden tomar distintas formas que determinan la Capacidad de Eslabonamiento Tecnológico (CET), necesaria para que la empresa pueda recibir y transmitir información, experiencias y tecnologías a lo interno y a lo externo de la empresa; $y$ fortalecer su accionar través de ello.

Una empresa podría no contar con todo el conocimiento, experiencia y capacidad para generar nuevas tecnologías, y en su lugar podría contar con una red de contactos externos o vínculos con las fuentes de información, de tecnologías, de buenas prácticas, de recursos humanos y financieros; para innovar (OCDE, 2005, 27). A este tipo de vínculo se le reconoce como eslabonamiento tecnológico.

Las carencias en materia de vinculaciones internacionales y las dificultades que experimenta la región latinoamericana para adaptarse a las nuevas reglas del juego por su mayor exposición relativa a las fallas del mercado y por sus más débiles estructuras productivas, administrativas y comerciales, han llevado a que las estrategias defensivas prevalezcan por sobre las iniciativas de producción y del dominio tecnológico, con un enfoque más estratégico.

Cada una de las capacidades antes descritas, que han sido identificadas en la literatura, posee una relevancia en los procesos de innovación, por lo que el análisis sistémico y holístico que identifiquen sus articulaciones e interrelaciones, resultan fundamentales en el enfoque del sistema de evaluación que se propone.

\section{Revisión de literatura sobre PYMES e innovación}

La conducta tecnológica empresarial, frente a la innovación, en la región latinoamericana es un tema que adolesce de suficiente información para realizar estudios en profundidad (Jaramillo et al., 2001, 1519). Desde el punto de vista de sus capacidades, las PYMES poseen limitaciones a la hora de innovar. Entre las razones se encuentran la falta de recursos y de know-how tecnológico lo que conlleva que la innovación se produzca de manera reactiva (Freel,
2000; Hassink, 1997; Smallbone et al., 2003). Este carácter reactivo y a corto plazo de la innovación dificulta la cooperación con agentes regionales de $\mathrm{I}+\mathrm{D}$ como universidades y centros tecnológicos. Entendiéndose por centros tecnológicos, espacios para el estudio, adaptación, generación y aplicación de nuevas tecnologías.

Se subraya la importancia de que las PYMES desarrollen competencias internas como condición indispensable para que puedan establecer relaciones fructíferas de cooperación con agentes externos y sean capaces de hacer uso de información externa e integrarla con la procedente del interior de la empresa (Kauffman \& Tödtling, 2002). En América Latina existen profundos interrogantes con respecto a las características y alcance de los procesos de cambio tecnológico a todos los niveles (REDES \& RICYT, 2008). En la práctica es difícil la generación de información concreta que responda a tales interrogantes; por lo que disponer de un sistema de evaluación de capacidades de innovación en PYMES vendría a solventar parte de esta falencia.

\section{Resultados y discusión}

\subsection{Aspectos conceptuales}

Para desarrollar el modelo se tomó como referente teórico el modelo interactivo del proceso de innovación (Kline \& Rosenberg, 1986; Rosenberg \& Nelson, 1994), en el cual se considera que por lo general, la empresa no dispone de los conocimientos científicos o técnicos que precisa para mejorar sus procesos o productos, por lo que acude a su entorno nacional o internacional a través de consultorías, investigaciones $\mathrm{u}$ otras formas de generación de conocimiento, de tal manera que la investigación y el desarrollo experimental $(\mathrm{I}+\mathrm{D})$ no se considera una fuente de innovación directa, sino una herramienta que se utiliza para resolver los problemas que aparecen en cualquier fase del proceso. Para contextualizar dicho modelo, se incorpora de manera explícita, una fase pre-innovación imprescindible en los procesos de innovación en $\mathrm{PED}$, en virtud de los esfuerzos previos que se realizan antes de concretarse una innovación incremental. En la Gráfica 2, se presenta el modelo conceptual propuesto para el análisis de la GI en PED. 
Gráfica 2. Modelo conceptual propuesto²

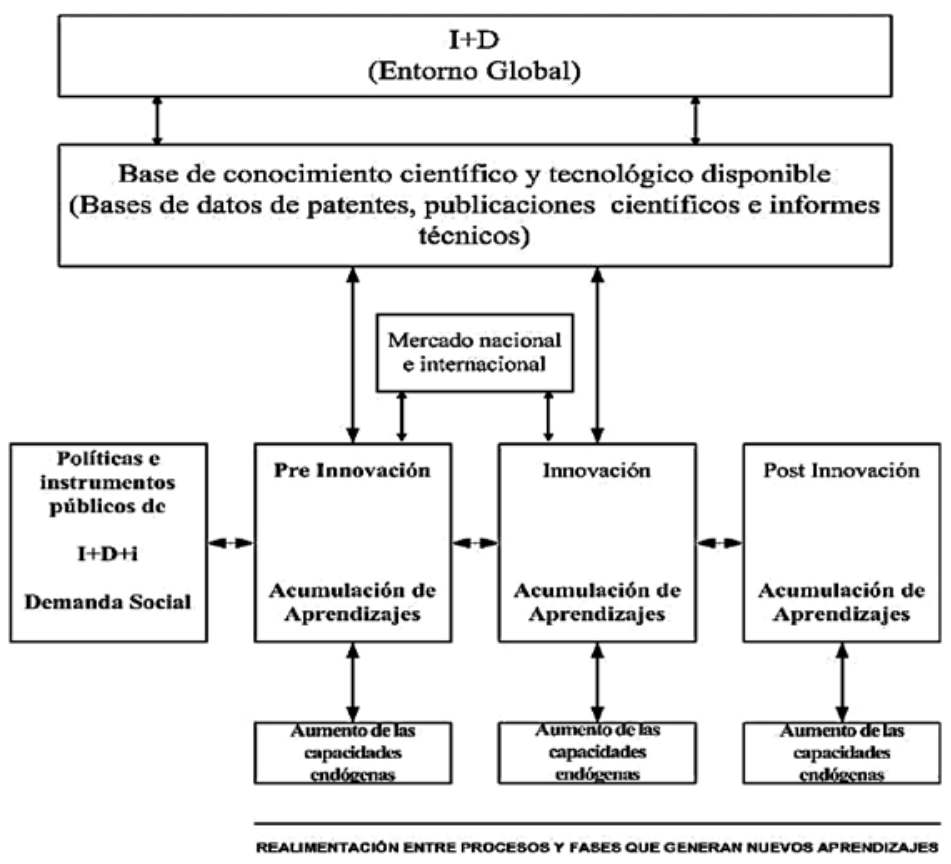

Para hacer funcional el modelo conceptual propuesto se hizo una adecuación de las funciones de GI plantadas por Morin (2001) y tras la comparación de estas funciones y el modelo TEMAGUIDE -elementos claves del proceso de innovación- (COTEC, 1998, 27), se plantea un modelo ajustado de GI para PED (Tabla 1).

Tabla 1. Comparativo de modelos ${ }^{3}$

\begin{tabular}{|c|c|c|}
\hline $\begin{array}{c}\text { Modelo } \\
\text { Tradicional } \\
\text { Morin (2001) }\end{array}$ & $\begin{array}{c}\text { Modelo } \\
\text { TEMAGUIDE } \\
\text { COTEC (1998) }\end{array}$ & $\begin{array}{c}\text { Modelo de } \\
\text { Gestión de la } \\
\text { Innovación (MGI) } \\
\text { Moreno (2013) }\end{array}$ \\
\hline Inventariar & ---- & Conocer \\
\hline Vigilar & Vigilar & ---- \\
\hline Focalizar & Focalizar & Focalizar \\
\hline---- & Capacitar / Aprender & Fortalecer \\
\hline Implantar & Implantar & Proteger \\
\hline Proceder & Proteger & Actuar \\
\hline
\end{tabular}

En el nuevo modelo MGI se incorporan dos nuevos elementos diferenciadores de procesos de innovación en PED. Los FPA (focos de procesos de aprendizajes) y los iones FII (focos de innovaciones incrementales).

Los FII son identificados en los procesos conocer $y$ focalizar (Tabla 1), los cuales dan lugar a proyectos $\mathrm{de} \mathrm{I}+\mathrm{D}+\mathrm{i}$ (actuar y obtener). Los FPA se generan en las interfaces entre procesos. El proceso fortalecer es transversal a los demás procesos. En la Gráfica 3 se muestra la relación del modelo MGI y los FPA.

\subsection{Sistema de evaluación de capacidades de innovación (SIGE V1)}

El modelo conceptual de MGI ha sido sustentado por modelos, métodos y referentes metodológicos validados por diferentes autores y experiencias en la región latinoamericana. Uno de los componentes del MGI es el módulo de PE (perfil empresarial) el cual

\footnotetext{
2 Fuente: Elaboración propia.

3 Fuente: Elaboración propia. Adaptación del Modelo COTEC (1998).
} 
Gráfica 3. Modelo MGl y su relación con los FPA ${ }^{4}$

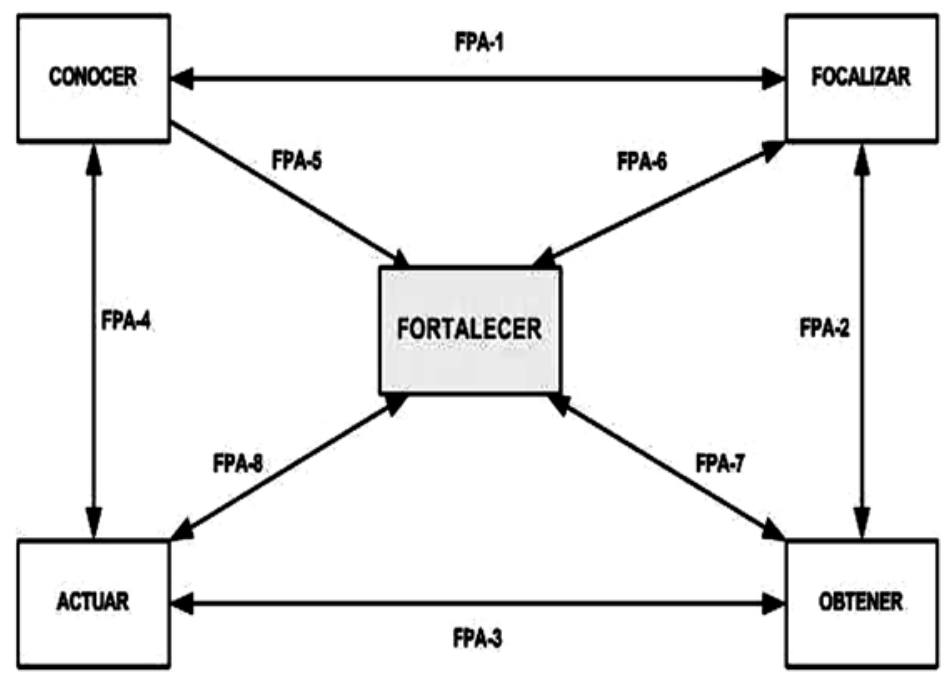

constituye la base metodológica del sistema de evaluación de capacidades endógenas propuesto.

Para determinar los descriptores e indicadores de cada capacidad considerada se analizaron y compararon entre sí, dos métodos y una metodología de evaluación de procesos de innovación en empresas latinoamericanas, basadas en la descripción y cuantificación de factores relacionados con la capacidad tecnológica y de innovación. El primer método es descriptivo para la caracterización de los procesos de innovación en la industria colombiana (Malaver \& Vargas, 2004); el segundo, constituye una propuesta específica de medición cuantitativa de la "capacidad innovativa" en una empresa (Yoguel \& Boscherini, 1996). El tercer modelo analizado, corresponde a una metodología de tipo cualitativa y descriptiva para evaluar la innovación, la tecnología y las competencias de una empresa (Delgado et al., 2008, 1-14).

La adaptación e integración de los aportes de los métodos y la metodología seleccionados constituye la base metodológica para la medición de las categorías de análisis identificadas en el capítulo anterior, para ello se realizó un estudio comparativo de los métodos.
Para cada capacidad de endógena, se identificaron sus correspondientes descriptores. Cada uno denota un requerimiento que la empresa debiera poseer. La valorización de las capacidades mediante éstos descriptores permite a la empresa conocer acerca de que tanto está en posibilidades de desarrollar con éxito procesos de innovación.

\subsubsection{Capacidad de gestión tecnológica (CGT)}

La CGT es un conjunto de prácticas utilizadas por la empresa para incorporar, explotar y desarrollar su patrimonio tecnológico, así como para vincularse y salvaguardar el conocimiento generado. La empresa debe detectar los cambios en tecnologías claves o líder del ámbito empresarial (aunque parezcan ligeros); lo que le facilitará el control continuo de los nuevos desarrollos tecnológicos en el entorno. Las técnicas de análisis de patentes son de mucha ayuda debido a que ofrecen información sobre senderos tecnológicos y oportunidades de indagar en nuevas tecnologías.

La CGT involucra 4 elementos (COTEC, 1998, 28): i) desarrollo de la estrategia tecnológica en la empresa, ii) adquisición de tecnologías, iii) desarrollo de nuevos productos, y iv) innovación de procesos.

4 Fuente: Elaboración propia. 
Estos elementos son incorporados de acuerdo a trece descriptores que fueron identificados a través del análisis realizado de los indicadores que se plantean en el Manual de Bogotá (Tabla 2).

Tabla 2. Descriptores para evaluar la CGT ${ }^{5}$

\begin{tabular}{|l|l|}
\hline \multicolumn{1}{|c|}{ Descriptor } & \multicolumn{1}{|c|}{ Descriptor } \\
\hline $\begin{array}{l}\text { 1. Existencia de estrategias em- } \\
\text { presariales relacionadas con la } \\
\text { adquisición, uso y evaluación de la } \\
\text { tecnología. }\end{array}$ & $\begin{array}{l}\text { 2. Existencia de una unidad encar- } \\
\text { gada de gestionar procesos de in- } \\
\text { novación en la organización. }\end{array}$ \\
\hline $\begin{array}{l}\text { 3. Grado de relación de la tecnología } \\
\text { con las estrategias empresariales. }\end{array}$ & $\begin{array}{l}\text { 4. Aplicación de técnicas para la se- } \\
\text { lección y valorización de tecnología. }\end{array}$ \\
\hline $\begin{array}{l}\text { 5. Grado de vínculo de las tecnolo- } \\
\text { gías adquiridas con las capacitacio- } \\
\text { nes del personal. }\end{array}$ & $\begin{array}{l}\text { 6. Grado de conocimiento del mer- } \\
\text { cado local. }\end{array}$ \\
\hline $\begin{array}{l}\text { 7. Grado de relación de la tecnolo- } \\
\text { gía con actividades de innovación } \\
\text { 0 desarrollo de nuevos productos, } \\
\text { servicios o procesos, cambios en la } \\
\text { organización, otros. }\end{array}$ & $\begin{array}{l}\text { 8. Esfuerzo para la introducción de } \\
\text { parámetros o estándares interna- } \\
\text { cionales de calidad (ingeniería de } \\
\text { producto, proceso, otros). }\end{array}$ \\
\hline $\begin{array}{l}\text { 9. Adopción de nuevas tecnologías } \\
\text { (un salto técnico 0 una transforma- } \\
\text { ción significativa de métodos 0 una } \\
\text { innovación incremental). }\end{array}$ & $\begin{array}{l}\text { 10. Inversión en capacitación tecno- } \\
\text { lógica o especializada para la gene- } \\
\text { ración de productos 0 servicios. }\end{array}$ \\
\hline $\begin{array}{l}\text { 11. Mecanismos para monitorear la } \\
\text { solución de los problemas de tipo } \\
\text { tecnológicos en la empresa. }\end{array}$ & $\begin{array}{l}\text { 12. Personal de la empresa capaci- } \\
\text { tado en el área de su trabajo. }\end{array}$ \\
\hline $\begin{array}{l}\text { 13. Aplicación de las TIC para ge- } \\
\text { nerar ideas o identificar oportuni- } \\
\text { dades. }\end{array}$ & \\
\hline
\end{tabular}

A través de la ecuación 1 se obtiene la valoración de la CGT, de acuerdo al promedio de valores (de uno a cuatro) dado por la empresa a cada descriptor.

$$
\text { CGT }=\sum \text { Val GT }(1 \text { a } 13) / 13
$$

Donde, Val GT corresponde a la valoración de uno a cuatro, dada por el informante de la PYME.

Son ocho los descriptores que fueron identificados para medir esta capacidad, los cuales se detallan en la Tabla 3.

Tabla 3. Descriptores para evaluar la CGA6

\begin{tabular}{|l|l|}
\hline \multicolumn{1}{|c|}{ Descriptor } & \multicolumn{1}{|c|}{ Descriptor } \\
\hline $\begin{array}{l}\text { 1.Registro de experiencias y resul- } \\
\text { tados durante el procesos de adop- } \\
\text { ción de tecnología. }\end{array}$ & $\begin{array}{l}\text { 2. Esfuerzos para garantizar la per- } \\
\text { manencia de conocimiento del } \\
\text { personal saliente (conocimiento } \\
\text { documentado). }\end{array}$ \\
\hline $\begin{array}{l}\text { 3. Existencia de mecanismos para } \\
\text { salvaguardar resultados de proce- } \\
\text { sos de innovación. }\end{array}$ & $\begin{array}{l}\text { 4. Acceso amplio en la empresa del } \\
\text { conocimiento que se genera de los } \\
\text { esfuerzos de innovación. }\end{array}$ \\
\hline $\begin{array}{l}\text { 5. Reconocimiento y recompensas } \\
\text { por el conocimiento creado y com- } \\
\text { partido dentro de la empresa. }\end{array}$ & $\begin{array}{l}\text { 6. Existencia de procedimientos } \\
\text { para identificar necesidades futuras } \\
\text { de conocimiento. }\end{array}$ \\
\hline $\begin{array}{l}\text { 7. Aplicación de la experimentación } \\
\text { como forma de aprendizaje. }\end{array}$ & $\begin{array}{l}\text { 8. Implementación de planes para } \\
\text { satisfacer las necesidades futuras } \\
\text { de conocimiento. }\end{array}$ \\
\hline
\end{tabular}

Por su parte, la valoración se realiza según la ecuación 2.

$$
\mathrm{CGA}=\sum \text { Val CGA }(1 \text { a } 8) / 8
$$

Donde, Val CGA corresponde a la valoración de uno a cuatro, dada por el informante de la PYME.

\subsubsection{Capacidad de asimilación de tecnología (CAT)}

Los diferentes planteamientos dejan en evidencia la importancia de que la empresa asimile las tecnologías adquiridas; es decir, uso diferenciado de la tecnología, ya que no solo debe adoptarla, sino más aun, adaptarla a sus requerimientos específicos y nuevos desafíos (OCDE, 2005). Se identifican siete descriptores (Tabla 4).

\footnotetext{
5 Fuente: Elaboración propia.
}

6 Fuente: Elaboración propia. 
Tabla 4. Descriptores para evaluar la CAT

\begin{tabular}{|l|l|}
\hline \multicolumn{1}{|c|}{ Descriptor } & \multicolumn{1}{|c|}{ Descriptor } \\
\hline $\begin{array}{l}\text { 1. Inversión en capacitación tec- } \\
\text { nológica especializada para utilizar } \\
\text { nuevas tecnologías adquiridas. }\end{array}$ & $\begin{array}{l}\text { 2. Utilización de mecanismos para } \\
\text { canalizar las ideas del personal téc- } \\
\text { nico sobre la mejorara del producto } \\
\text { 0 servicio. }\end{array}$ \\
\hline $\begin{array}{l}\text { 3. Interacción con diferentes pro- } \\
\text { veedores o competidores. }\end{array}$ & $\begin{array}{l}\text { 4. Respuesta del personal frente a } \\
\text { los problemas tecnológicos ines- } \\
\text { perados. }\end{array}$ \\
\hline $\begin{array}{l}\text { 5. Utilización de mecanismos para } \\
\text { que el personal aporte nuevas ideas } \\
\text { que mejoren el producto 0 servicio. }\end{array}$ & $\begin{array}{l}\text { 6. Participación de la empresa en } \\
\text { proyectos de innovación concluidos }\end{array}$ \\
\hline $\begin{array}{l}\text { 7. Participación de la empresa en } \\
\text { proyectos de I+D+i. }\end{array}$ & \multicolumn{2}{|l}{} \\
\hline
\end{tabular}

Para medir la CAT se plantea la ecuación 3.

$$
\mathrm{CAT}=\sum \text { Val CAT }(1 \text { a } 7) / 7
$$

Donde, Val CAT corresponde a la valoración de uno a cuatro, dada por el informante de la PYME.

\subsubsection{Capacidad de eslabonamiento tecnológico (CET)}

Tabla 5. Descriptores para obtener el valor de GVT $(60 \%)^{8}$

\begin{tabular}{|l|l|}
\hline \multicolumn{1}{|c|}{ Descriptores } & \multicolumn{1}{c|}{ Descriptores } \\
\hline $\begin{array}{l}\text { 1. Existencia de mecanismos de } \\
\text { vínculos para realizar alianzas. }\end{array}$ & $\begin{array}{l}\text { 2. Asociatividad de la empresa en } \\
\text { el sector de actividad económica } \\
\text { nacional o internacional. }\end{array}$ \\
\hline $\begin{array}{l}\text { 3. Participación de la empresa en } \\
\text { proyectos de I+D con otras insti- } \\
\text { tuciones. }\end{array}$ & $\begin{array}{l}\text { 4. Vínculos y relaciones con otros } \\
\text { agentes del Sistema de Ciencia, } \\
\text { Tecnología e Innovación del país. }\end{array}$ \\
\hline $\begin{array}{l}\text { 5. Acercamiento con Centros de } \\
\text { I+D nacionales o internacionales. }\end{array}$ & $\begin{array}{l}\text { 6. Existencias de normas o pau- } \\
\text { tas que rigen las interrelaciones } \\
\text { tecnológicas cn otros agentes del } \\
\text { entorno. }\end{array}$ \\
\hline
\end{tabular}

La valoración de la CET se obtiene a partir de dos componentes. Uno que determina la gestión del vínculo tecnológico (GVT) y el otro que determina la intensidad del vínculo tecnológico (IVT). A estos componentes se les ha asignado un peso de acuerdo su aporte en la gestión del aprendizaje (Moreno, 2013). De esta manera el peso asignado para el GVT es de 0,60 (Tabla 5) y el del IVT es de 0,40 (ecuación 4).

$$
\text { GVT } \left.=\sum \text { Val GVT (1 a } 6\right) / 6
$$

La intensidad de vinculación (IVT) responde a la ecuación 5 .

$$
\text { IVT }=0,20 *(\text { Val IVI })+0,80 *(\text { IVF-CET })
$$

f ( 1 a 8) son los valores de los factores. Las áreas temáticas son establecidas según el contexto.

Val IVI es la intensidad de vinculación informal y se calcula de acuerdo con la ecuación 6.

$$
\text { Val IVI }=\sum[\mathrm{f} 1 * 0,30+\mathrm{f} 2 * 0,30+\mathrm{f} 3 * 0,20+\mathrm{f} 4 * 0,20](6)
$$

Val IVF es la intensidad de vinculación formal y se calcula de acuerdo con la ecuación 7 .

$$
\mathrm{IVF}=\sum[\mathrm{f} 5+\mathrm{f} 6+\mathrm{f} 7+\mathrm{f} 8](7)
$$

Tabla 6. Descriptores para obtener el IVT ${ }^{9}$

\begin{tabular}{|l|l|}
\hline \multicolumn{1}{|c|}{$\begin{array}{c}\text { Descriptor para ponderar } \\
\text { IVI (peso = 20\%) }\end{array}$} & $\begin{array}{c}\text { Descriptor para ponderar } \\
\text { IVF (peso = 80\%) }\end{array}$ \\
\hline $\begin{array}{l}\text { 1. Frecuencia de vínculos informa- } \\
\text { les. }\end{array}$ & $\begin{array}{l}\text { 1. Número de agentes con los que } \\
\text { se mantiene vínculo. }\end{array}$ \\
\hline $\begin{array}{l}\text { 2. Complejidad de las áreas temá- } \\
\text { ticas en las que se mantiene vín- } \\
\text { culos. }\end{array}$ & 2. Complejidad de la interacción. \\
\hline $\begin{array}{l}\text { 3. Número de agentes con los que } \\
\text { se mantiene vínculos. }\end{array}$ & 3. Frecuencia de la interacción. \\
\hline $\begin{array}{l}\text { 4. Estabilidad de los vínculos in- } \\
\text { formales: refleja el nivel alcanzado } \\
\text { según los objetivos de la empresa } \\
\text { en su interacción con otros agentes. }\end{array}$ & $\begin{array}{l}\text { 4. Vínculos. } \\
\text { por la la firma de un documento con- } \\
\text { tractual. }\end{array}$ \\
\hline
\end{tabular}

Finalmente la relación queda tal como se presenta en la ecuación 8.

$$
\mathrm{CET}=(0,60 * \mathrm{GVT})+(0,40 * \operatorname{IVT})(8)
$$

\footnotetext{
Fuente: Elaboración propia.

Fuente: Elaboración propia.

Fuente: Elaboración propia.
} 


\subsection{Aplicación del sistema y análisis de resultados}

- La confiabilidad de los resultados fue posible al contar con casos de estudios con situaciones reales. Hubo buena disposición en suministrar la información y los resultados pueden ser aplicados a nuevos casos de estudio en contextos de PED.

- Los instrumentos de recolección de información resultaron efectivos en las mediciones que se estipularon. La validez externa estuvo dada por las condiciones homologadas durante el proceso de recolección de información. El cual tuvo lugar en dos momentos diferentes. En la fase inicial se abordaron los cuatro casos de estudio, a quienes se les aplicaron los cuestionarios y las entrevistas en iguales condiciones. En la segunda fase, correspondiente a la verificación de los componentes funcionales hubo dos empresas que no lograron continuar con el proceso final, debido a situaciones internas de las propias empresas.

- La evaluación de capacidades (primera fase) fue obtenida a partir de la auto valoración de las empresas de indicadores y descriptores, mediante una escala Richter de 1 a 4 ( 1 = baja, nunca, ninguno; 4 = alto, siempre, alguno). Para lo cual se dieron instrucciones precisas y se suministró información conceptual y operacional a los 4 casos de PYME seleccionadas (Tabla 7).

Tabla 7. Descripción de casos de estudio ${ }^{10}$

\begin{tabular}{|c|c|c|c|}
\hline Caso & $\begin{array}{c}\text { Sector } \\
\text { económico }\end{array}$ & $\begin{array}{c}\text { Total de } \\
\text { empleados }\end{array}$ & Lugar \\
\hline 1 & TIC/Servicios & 25 & Provincia Panamá \\
\hline 2 & Agroindustria & 6 & Provincia Coclé \\
\hline 3 & Agropecuario & 7 & Provincia Chiriquí \\
\hline 4 & Producción Industrial & 7 & Provincia Panamá \\
\hline
\end{tabular}

- Se utilizaron plantillas pre-elaboradas para presentar la valoración de los descriptores de capacidades endógenas de los casos de estudio.

- Se aplicaron las fórmulas para obtener las valoraciones promedios de cada descriptor $e$ indicador.

- Los resultados de las capacidades endógenas se presentan en la Tabla 8.

El trabajo de campo permitió:

- Constatar el bajo nivel de las capacidades endógenas (Tabla 8).

Tabla 8. Evaluación de las capacidades endógenas ${ }^{11}$

\begin{tabular}{|c|c|c|c|c|c|c|c|c|}
\hline \multirow{2}{*}{ Capacidad } & \multicolumn{2}{|c|}{ CASO 1 } & \multicolumn{2}{c|}{ CASO 2 } & \multicolumn{2}{c|}{ CASO 3 } & \multicolumn{2}{c|}{ CASO 4 } \\
\cline { 2 - 9 } & Valor & $\begin{array}{c}\text { Nivel de la } \\
\text { capacidad }\end{array}$ & Valor & $\begin{array}{c}\text { Nivel de la } \\
\text { capacidad }\end{array}$ & Valor & $\begin{array}{c}\text { Nivel de la } \\
\text { capacidad }\end{array}$ & Valor & $\begin{array}{c}\text { Nivel de la } \\
\text { capacidad }\end{array}$ \\
\hline CGT & 3,07 & Alta & 1,85 & Baja & 3,07 & Alta & 2,08 & Media \\
\hline CGA & 2,75 & Media & 2,00 & Baja & 3,25 & Alta & 1,88 & Baja \\
\hline CAT & 1,86 & Baja & 2,71 & Media & 3,43 & Alta & 2,14 & Media \\
\hline CET & 2,99 & Media & 2,43 & Media & 2,71 & Media & 2,36 & Media \\
\hline
\end{tabular}

10 Fuente: Elaboración propia.

11 Fuente: Elaboración propia. 
- Comparar el patrón de comportamiento teórico esperado según el perfil empresarial (PE) de cada caso y las capacidades endógenas con el patrón real. Para lo cual se examinó si los datos recogidos convergen hacia una secuencia lógica de acontecimientos para una explicación tentativa de acuerdo a la sucesión hipotética de eventos a identificar.

- Verificar la relación entre niveles de capacidades y el perfil empresarial en cada caso. Cons- tando que en empresas más innovadoras, las capacidades endógenas obtienen una mayor valoración. Por ejemplo, los valores de las capacidades CET y CGT del Caso 1 junto con su RI son iguales a los del Caso 3, lo que muestra que una intensidad en actividades innovativas, medida de acuerdo a los resultados intermedios (RI) obtenidos por la empresa, podría aproximarla a una inminente innovación de producto o servicio (Gráfica 5).

Gráfica 5. Comparación global de la valoración de las categorías de análisis ${ }^{12}$

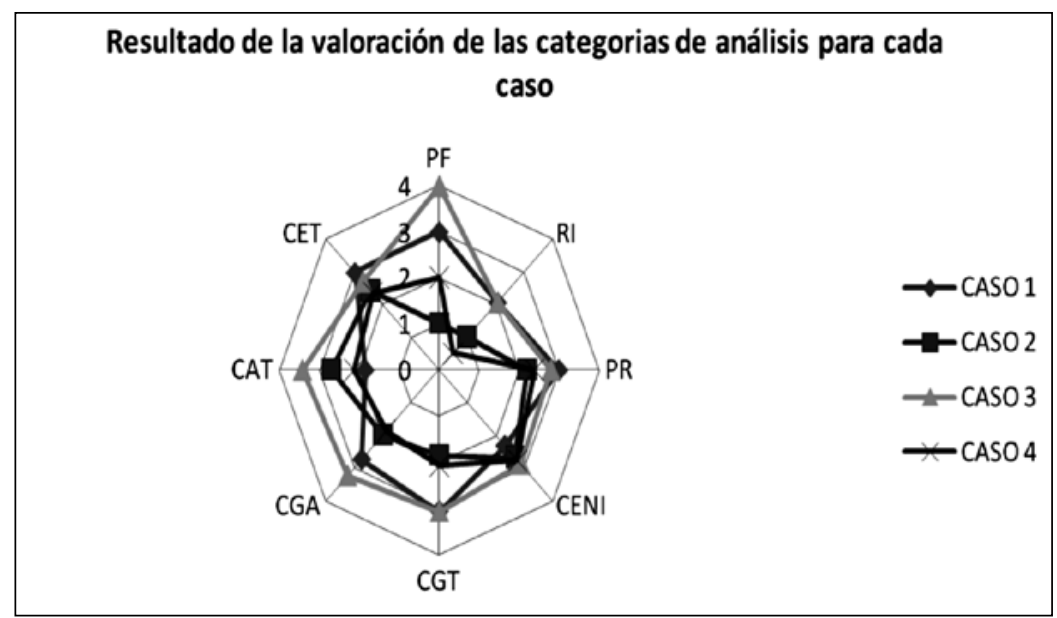

De manera complementaria, el análisis más profundo de dos de los casos de estudio permitió puntualizar lo siguiente:

- El conocimiento específico sobre su nivel de capacidades endógenas les ha motivado a elaborar, con el apoyo de expertos, un plan de fortalecimiento de los aspectos que describen cada capacidad evaluada.

- Se visualiza con mayor claridad la importancia de indagar más a fondo con los procesos formales de gestión de innovación, hasta ahora se ha manejado de manera algo informal, aunque pareciera haber obtenido buenos resultados.

- La CGT estará caracterizada por un mayor enfoque estratégico y formalización en la gestión de la tecnología adoptada o adaptada por la empresa. Mientras que la CGI aumentará en la medida en que aumente CET, CGA y CAT (Gráfica 6). Este planteamiento no implica una realización lineal de las funciones de innovación, sino más bién, una interrelación entre ellas. El énfasis del enfoque está en el desarrollo de las capacidades y de los esfuerzos que se realizan para innovar.

12 Fuente: Moreno (2013). 
Gráfica 6. Interrelación entre capacidades ${ }^{13}$

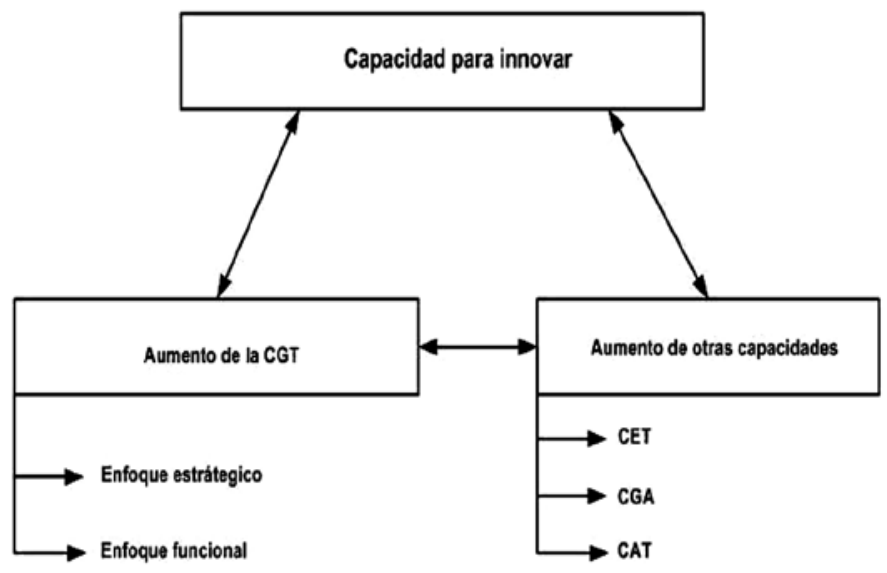

\section{Conclusiones}

- El modelo conceptual propuesto se constituye en un fundamento válido de gestión de aprendizaje para los procesos de evaluación de innovación.

- Los aportes de los métodos evaluados resultaron ser útiles para determinar una métrica de evaluación de capacidades endógenas.

- La evaluación basada en capacidades aborda el tema de la innovación desde una perspectiva de la realidad específica de la empresa y de la especificidad que se requiere para la eficacia en el esfuerzo hacia la competitividad empresarial basada en la innovación.

- Se propone a las PYME una forma sistemática de analizar y fortalecer sus capacidades y procesos gerenciales, contrarrestando, la informalidad y la falta de información para la toma de decisiones.

- La ampliación de la base conceptual de procesos de innovación, con la introducción a las categorías de análisis dos nuevos conceptos: FII y FPA, permite realizar una evaluación más precisa de las capacidades de la empresa.
- El estudio de las capacidades para innovar permite profundizar en el ámbito funcional de la empresa lográndose identificar procesos de aprendizajes (FPA) y posibles innovaciones incrementales (FII), que se pueden constituir en hoja de ruta para una empresa en post de una mayor competitividad.

- El conocimiento específico sobre el nivel de capacidad para innovar ha motivado a elaborar, con el apoyo de expertos, un plan de fortalecimiento de los aspectos que describen cada capacidad evaluada (hoja de ruta) para innovar más.

\section{Referencias}

Angelelli, P. \& Gligo, N. (2002). Apoyo a la innovación tecnológica en América Central: la experiencia del fondo para la Modernización Tecnológica y Empresarial de Panamá. Washington DC: BID.

Bell, M. \& Pavitt, K. (1995). Diagnóstico de capacidades. CEPAL.

Castro, J., Roca, L. \& Ibarra, L. (2009). Capacidad de absorción y formas de aprendizaje para la innovación: un modelo conceptual. En: Projectics/Proyéctica/Projectique, De Boeck Supérieur, 1(1): 63-76.

CEPAL (2010). Desarrollo social de América Latina. Santiago de Chile.

Cohen, W. \& Levinthal D. (1990). Absorptive capacity: a new perspective of learning and innovation. En: Administrative Science Quarterly, Social Science Research Network, 35(1): 128-152.

COTEC (1998). TEMAGUIDE. Pautas metodológicas en gestión de la tecnología y de la innovación para empresas. Tomos №1, 2 y 3. España.

13 Fuente: Elaboración propia. 
Delgado, M., Pino, J., Solís, F. \& Barea, M. (2008). Evaluación integrada de la innovación, la tecnología y las competencias en la empresa. En: Revista Madrid, Universidad Autónoma de Madrid, Tribuna de debate (47): 1-14.

Dini, M. \& Stumpo, G. (2004). Pequeñas y medianas empresas y eficiencia colectiva. Estudios de caso en América Latina, Siglo XXI-CEPAL, México

Figueiredo, L. (2001). Latin America transformed-globalization and modernity. En: Journal of International Development, Wiley Online Library, 13(2): 289-290.

Freel, M. (2000). Strategy and structure in innovative manufacturing SMEs: the case of an english region. En: Small Business Economics, Springer, 15(1): 27-45.

Hassink, R. (1997). Technology transfer infrastructures: some lessons from experiences in Europe, the US and Japan. En: European Planning Studies, Taylor \& Francis, 5(3): 351-370.

Hidalgo, A., León, G. \& Pavón, J. (2000). La gestión de la innovación y la tecnología en las organizaciones. Madrid: Pirámide.

Jaramillo, H., Lugones, G. \& Salazar, M. (2001). Normalización de indicadores de innovación tecnológica en América Latina y el Caribe. Manual de Bogotá. Editado por RYCIT / OEA / CYTED / COLCIENCIAS/OCYT.

Kline, S. \& Rosenberg, N. (1986). An overview of innovation, in the positive sum strategy: harnessing technology for economic growth. Washington, D. C.: National Academy Press.

Lall, S. (1992). Technological capabilities and industrialization. En: World Development, Elsevier, 20(2): 165-186.

Lundvall, B. (1992). National Systems of Innovation - Toward a Theory of Innovation and Interactive Learning. Londres: Printer Publishers.

Malaver, F. \& Vargas, M. (2004). Hacia una caracterización de los procesos de innovación en la industria colombiana: los resultados de un estudio de caso. En: Revista Latinoamericana de Administración, CLADEA, 33

Moreno, S. (2013). Sistema para la gestión de innovación en países en desarrollo, basado en la autoevaluación integrada de capacidades endógenas. Articulación de la base conceptual del sistema experto para la autoevaluación. Ph. D. Thesis. Barcelona: Univesitat Politecnica de Catalunya.

Morin, J. (2001). Gestión de innovación, GETEC, ETSIT. La dirección estratégica de la empresa. Teoría y aplicaciones. Madrid: Civitas.

Nelson, R. (1993). National innovation systems: a comparative analysis. Oxford Univ. Press, New York. USA.

Nelson, R. (1997). Understanding technical change as an evolutionary process. North Holland. Elsevier Science Publishers, USA

OCDE (2005). Guía para la recogida e interpretación de datos sobre innovación (Manual de OSLO). Francia: Comisión Europea.

Pavitt, K. (1991). ¿Dónde reside la utilidad económica de la investigación básica? En: Arbor: Ciencia, Pensamiento y Cultura, Servicio de publicaciones del CSIC, 139(546): 31-56.

Porter, M. (1998). Clusters and the new economics of competition. En: Harvard Business Review, Harvard College, 76(6): 77-90.

RAE (1992). Diccionario de la Lengua Española. Tomo III, Edición 21. Madrid: Espasa Calpe.

REDES \& RICYT (2008). El estado de la ciencia: principales indicadores de ciencia y tecnología Iberoamericanos/Interamericanos. Buenos Aires: RICYT.

Rosenberg, N. \& Nelson, R. (1994). American universities and technical advance in industry. Washington, D. C.: National Academy Press.

Smallbone, D., North, D. \& Vickers, I. (2003). Part I. SMEs, innovation and regions: conceptual background. The role and characteristics of SMEs. En: Asheim, B., Isaksen, A., Nauwelaers, C. \& Tödtling, F. (eds.) Regional innovation policy for smallmedium enterprises. Cheltenham: Edwar Elgar.

Schumpeter, J. (1939). The theory of economic development. Harvard. USA.

Yoguel, G. \& Boscherini, F. (1996). La capacidad innovativa y el fortalecimiento de la competitividad de las firmas: el caso de las PyME exportadoras argentinas. En: Documentos de trabajo, CEPAL (71): 1-96. 